\title{
Heparinase selectively sheds heparan sulphate from the endothelial glycocalyx
}

\author{
Daniel Chappell1,2, Matthias Jacob ${ }^{1,2}$, Markus \\ Rehm ${ }^{1}$, Mechthild Stoeckelhuber ${ }^{3}$, Ulrich \\ Welsch ${ }^{3}$, Peter Conzen ${ }^{1}$ and Bernhard F. \\ Becker ${ }^{2, *}$
}

${ }^{1}$ Clinic of Anaesthesiology, Ludwig Maximilians

University, Nussbaumstr. 20, D-80336 Munich, Germany

${ }^{2}$ Institute of Physiology, Ludwig Maximilians University,

Schillerstr. 44, D-80336 Munich, Germany

${ }^{3}$ Institute of Anatomy, Ludwig Maximilians University,

Pettenkoferstr. 11, D-80336 Munich, Germany

${ }^{\star}$ Corresponding author

e-mail: b.f.becker@Irz.uni-muenchen.de

\begin{abstract}
A healthy vascular endothelium is coated by the endothelial glycocalyx. Its main constituents are transmembrane syndecans and bound heparan sulphates. This structure maintains the physiological endothelial permeability barrier and prevents leukocyte and platelet adhesion, thereby mitigating inflammation and tissue oedema. Heparinase, a bacterial analogue to heparanase, is known to attack the glycocalyx. However, the exact extent and specificity of degradation is unresolved. We show by electron microscopy, immunohistological staining and quantitative measurements of the constituent parts, that heparinase selectively sheds heparan sulphate from the glycocalyx, but not the syndecans.
\end{abstract}

Keywords: coronary system; endothelium; heart; permeability barrier; syndecans.

A healthy vascular endothelium is coated by a variety of transmembrane syndecans and membrane-bound glypicans, both containing heparan sulphate and chondroitin sulphate side chains, which, together with many shorter membrane proteins, constitute the endothelial glycocalyx (Pries et al., 2000; Rehm et al., 2004). The most common glycosaminoglycan on the endothelial glycocalyx is heparan sulphate (Bernfield et al., 1999; Pries et al., 2000). Principal proteins that carry bound heparan sulphate - the proteoglycans - are the transmembrane syndecans. The most prevalent proteoglycan on the vascular endothelium is syndecan-1 (Pries et al., 2000; Alexopoulou et al., 2007). It is well recognised that enzymatic digestion with heparinase, a bacterial analogue to heparanase, or with pronase, as well as exposure to tumour necrosis factor- $\alpha$ (TNF- $\alpha)$, ischaemia or major surgery can reduce the thickness of the endothelial glycocalyx, deteriorating the vascular barrier (Henry and Duling, 2000; Rehm et al., 2004).
In vivo, the glycocalyx binds plasma proteins, forming the endothelial surface layer. This has been demonstrated as an exclusion zone, e.g., for circulating erythrocytes (Pries et al., 2000; Pries and Kuebler, 2006). To date, there has been no direct visualisation of the glycocalyx in humans, mainly due to the fact that the structure is very fragile and easily deteriorates (Pries et al., 2000). Therefore, one possibility to investigate the state of the glycocalyx is to measure retention or shedding of its constituent parts, e.g., heparan sulphate, syndecan-1 or hyaluronan (Rehm et al., 2001; Nieuwdorp et al., 2006). We chose to investigate the effect of heparinase on heparan sulphate and syndecan-1, as hyaluronan has been reported only to be part of the endothelial surface layer and not of the glycocalyx itself (Pries and Kuebler, 2006). Here we show, for the first time, visually and quantitatively, that heparinase selectively sheds heparan sulphate, but not syndecan-1 off the glycocalyx in an intact vascular bed, namely the coronary system of the isolated perfused guinea-pig heart. This may help to elucidate the role of heparan sulphate in the regulation of vascular properties (Rehm et al., 2004; Jacob et al., 2006, 2007).

Guinea-pig hearts were isolated and perfused at constant coronary flow rate in a modified Langendorff mode, as described previously (Rehm et al., 2004; Jacob et al., 2006). Coronary venous effluent was collected from the cannulated pulmonary artery after draining from the coronary sinus into the right atrium and ventricle. After an equilibration time of $15 \mathrm{~min}$ to establish steady-state conditions, enzymatic degradation of the glycocalyx was performed by infusing heparinase, $10 \mathrm{U}$ of enzyme (heparinase type I; Sigma-Aldrich, Seelze, Germany) being applied into the intact coronary system in the course of $10 \mathrm{~min}$. Concentrations of heparan sulphate and syndecan-1 in the coronary effluent were determined using an ELISA (Seikagaku Corporation, Tokyo, Japan) or an enzyme-linked immunosorbent assay (Diaclone Research, Besancon, France), respectively. To visualise the glycocalyx with the electron microscope, hearts were perfusion-fixed at the end of the protocol via the aorta and coronary arteries with a solution containing $2 \%$ glutaraldehyde, $2 \%$ sucrose, $0.1 \mathrm{M}$ sodium cacodylate phosphate, and $2 \%$ lanthanum nitrate (Vogel et al., 2000). Lanthanum is a trivalent cation and binds to negatively charged glycoprotein moieties of the glycocalyx, stabilising the structure during fixation. For light microscopy and immunohistochemistry, the hearts were perfusion fixed with $4 \%$ formaldehyde and then stored in $1 \%$ formaldehyde solution for $24 \mathrm{~h}$. Paraffin sections $(5 \mu \mathrm{m})$ were stained with a monoclonal antibody against heparan sulphate (Seikagaku Corporation). Comparisons involving two groups were made using the Wilcoxon test for dependent data. A $p$-value of $<0.05$ was considered to be statistically significant. The statistical software used 
to conduct the analyses was SigmaStat 3.5 (Systat Software Inc., San Jose, CA, USA).

Small amounts of heparan sulphate and syndecan-1 were detectable in the coronary effluent under basal conditions. Basal rates of release were $3.2 \pm 1.0 \mu \mathrm{g} / \mathrm{min} / \mathrm{g}$ and $0.14 \pm 0.02 \mu \mathrm{g} / \mathrm{min} / \mathrm{g}$, respectively (Figure 1). During application of heparinase (10 $\mathrm{min})$, the rate of syndecan1 release amounted to $0.12 \pm 0.24 \mu \mathrm{g} / \mathrm{min} / \mathrm{g}$ in the last 5 $\mathrm{min}$, while that of heparan sulphate rose to $30.3 \pm 4.1$ $\mu \mathrm{g} / \mathrm{min} / \mathrm{g}$. Thus, syndecan- 1 concentrations showed no difference $(W=-12, \quad p>0.05)$, but highly significant amounts of heparan sulphate were shed from the glycocalyx ( $\min 0-5: W=-28, p=0.01$ ). Shedding was maintained, albeit at a lower level, for approximately $15 \mathrm{~min}$ during washout of the enzyme (Figure 1).

Electron microscopy showed a dramatic degradation of the endothelial glycocalyx (from approximately $220 \mathrm{~nm}$ to $20 \mathrm{~nm}$ ) after application of heparinase (Figure 2). Light microscopy and immunohistochemistry revealed heparan sulphate-positive staining of endothelial cells of small vessels and large arteries of the guinea-pig heart (Figure $3 A, C)$. Also, the walls of the vasculature contained heparan sulphate. After application of heparinase, the staining of the endothelial cell lining was markedly reduced in all types of vessels (Figure 3B,D). Thus, shedding of heparan sulphate off the endothelial surface is induced by heparinase. Enzymograms (PAGE, gelatine) showed that heparinase has no collagenase activity vs. control, excluding enzyme activity, e.g., against the basement

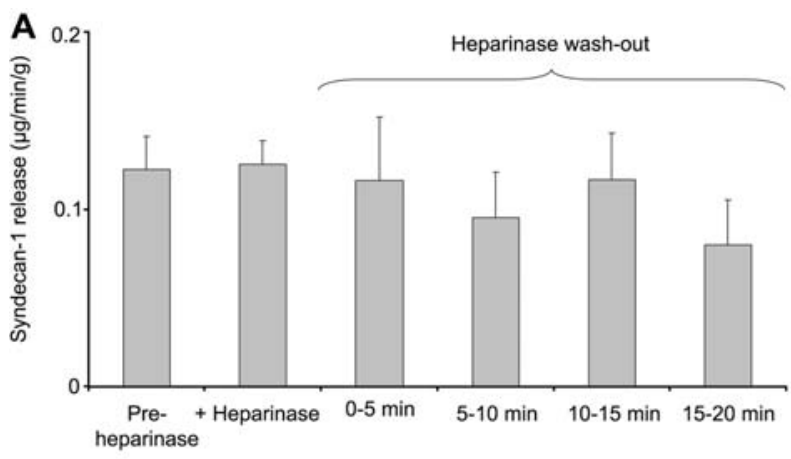

B

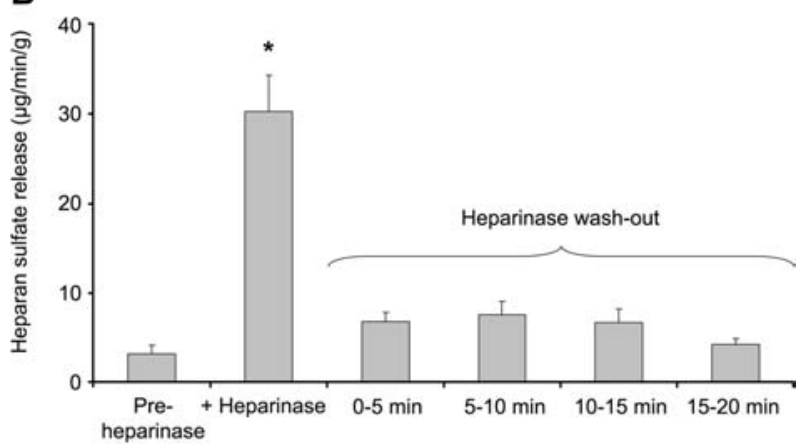

Figure 1 Coronary washout of constituents of the endothelial glycocalyx.

Release rates of syndecan-1 (top panel) and heparan sulphate (bottom panel) in the coronary effluent before, during and after treatment with heparinase (10 IU, $10 \mathrm{~min})$. Values are mean \pm SD of six hearts/group. ${ }^{*} p=0.01$ and ${ }^{*} p<0.05+$ heparinase vs. preand post-heparinase, respectively. membrane. This speaks against any substantial nonspecific protease activity of the applied enzyme.

Until today, the exact structure and function of the vascular endothelial glycocalyx is still uncertain. For many years, it was known as a layer with a thickness of only a few tens of nanometres (Luft, 1966). Over the past years, several animal studies have suggested the glycocalyx to be much thicker and to be involved in a substantial number of physiological and pathophysiological processes. Especially, it plays a major role in the vascular barrier function (Rehm et al., 2004; Jacob et al., 2006, 2007). Together with bound plasma proteins and solubilised glycosaminoglycans, the glycocalyx forms the endothelial surface layer with a thickness of approximately $1 \mu \mathrm{m}$ (Pries et al., 2000). The glycocalyx is also composed of membrane-bound molecular components, such as cell adhesion molecules, e.g., integrins and selectins (Pries et al., 2000), and binds and intercalates inflammatory cytokines and components of the coagulation system, e.g., tissue factor and fibrinogen (Shih and Hajjar, 1993). As the endothelial surface layer constitutes the first contact surface between blood and tissue, it is essentially involved in processes such as inflammation, coagulation and firm adhesion of leukocytes and platelets to the vascular wall. The importance of the endothelial glycocalyx in situ has been clearly shown after its perturbation in various animal studies (Henry and Duling, 2000; Rehm et al., 2004; Bruegger et al., 2005; Jacob et al., 2006).

There are no data available as to the possible physiological regulation of the glycocalyx or the rate of recovery after damage. However, endothelial caveolae are densely packed with glycocalyx, which might represent a reservoir for deployment on the endothelial surface (Jacob et al., 2007). Moreover, we believe that the glycocalyx will be more susceptible to deterioration if the endothelial surface layer is altered. As this mainly consists of intercalated plasma proteins and hyaluronan and is not anchored in the endothelial membrane, it may be able to re-organise itself much more easily than the glycocalyx.

Diminution of the glycocalyx leads to activation of coagulation, leukocyte and platelet adhesion and increases capillary permeability, leading to tissue oedema (van den Berg et al., 2003). Furthermore, ischaemia/ reperfusion impairs shear-stress mediated, endothelium (nitric oxide)-dependent coronary vasodilatation, contributing to low reflow, and leads to damage of the coronary endothelium (Bouchard and Lamontagne, 1999). In isolated heart preparations, as used here, ischaemia/ reperfusion was found to increase shedding of heparan sulphate approximately 30-fold (Chappell et al., 2006). In contrast, washout of hyaluronan was increased only 3fold above basal, even under such extreme conditions (unpublished results). Surgical stress is believed to deteriorate the endothelial glycocalyx to some degree, depending on the severity of the trauma. Indeed, manifestation of endothelial injury consists of a disruption of the glycocalyx. As illustrated in the present work, the tendency for syndecan-1 release in the venous effluent of isolated hearts to decrease with time might result from a recovery of the coronary endothelium after the surgical preparation. 


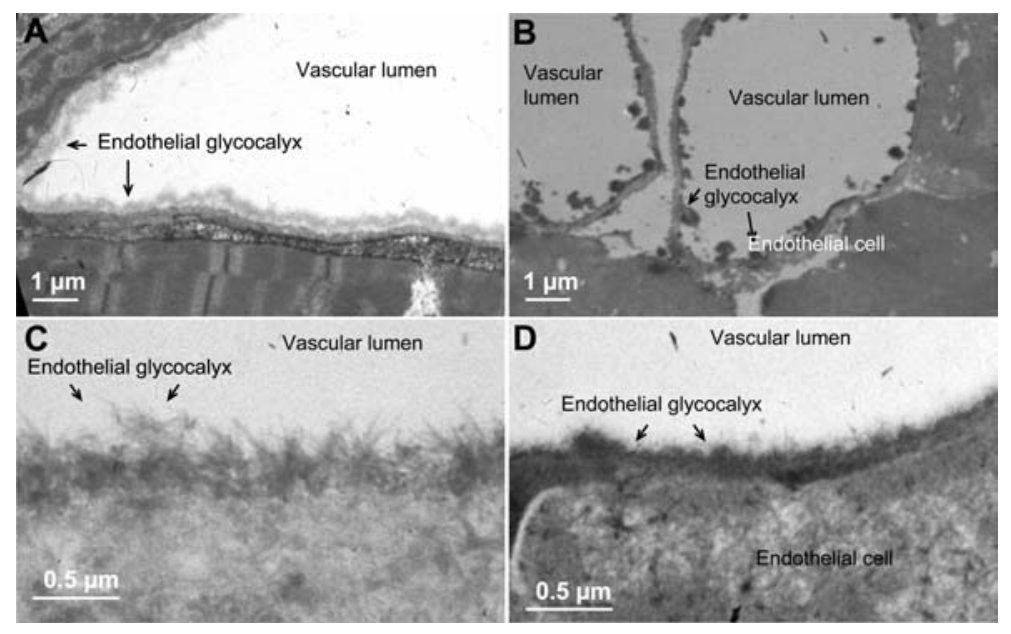

Figure 2 Electron micrographs of the endothelial glycocalyx in isolated perfused hearts (guinea-pig).

An intact glycocalyx is shown in an untreated control heart: panel (A) (overview) and panel (C) (close-up). After application of heparinase, the glycocalyx was deteriorated: panel (B) (overview) and panel (D) (close-up).

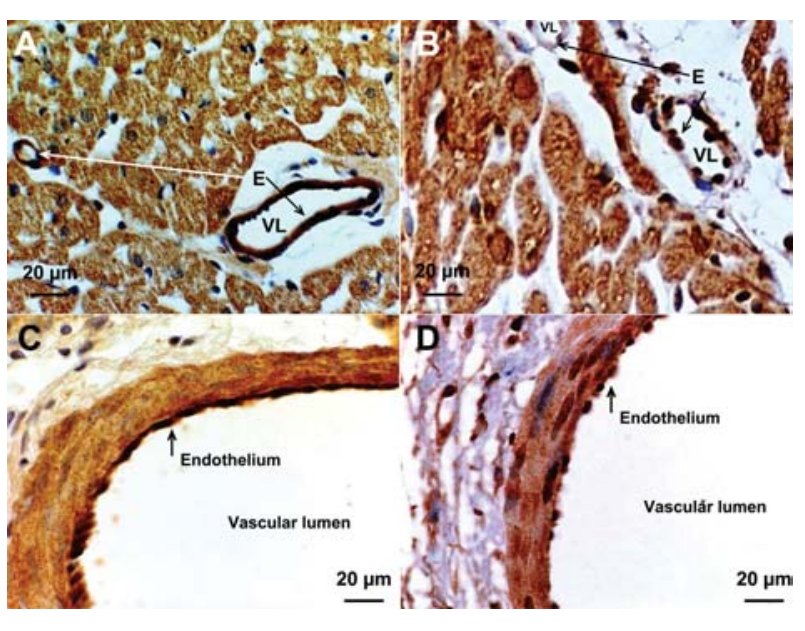

Figure 3 Immunostaining of coronary microvessels (panels $A$ and $\mathrm{B}$ ) and coronary artery (panels $\mathrm{C}$ and $\mathrm{D}$ ) with a monoclonal antibody against heparan sulphate.

Guinea-pig hearts were perfused without heparinase (panels A and $C$ ) and with heparinase (panels $B$ and D). The original magnification was $60 \times$ in all cases. E: endothelium; VL: vascular lumen.

Heparinase is a bacterial analogue to heparanase, a mammalian endoglycosidase participating in degradation and remodelling of the extracellular matrix and facilitating cell invasion associated with inflammation or cancer metastasis (Myler and West, 2002). In how far shedding of merely the charged heparan sulphate side-groups contributes to these pathophysiological phenomena in vivo remains to be determined.

In conclusion, heparinase specifically induces shedding of heparan sulphate from the glycocalyx, thereby deteriorating the vascular barrier, while syndecan-1 remains on the endothelial surface. This scaffolding seems to 'collapse', forming a thin layer of only one-tenth of the original thickness at the endothelial surface. As shown previously, such a rudimentary glycocalyx loses much of its ability to mediate shear stress to the endo- thelial cells and to act as a second barrier against vascular leakage.

\section{References}

Alexopoulou, A.N., Multhaupt, H.A., and Couchman, J.R. (2007). Syndecans in wound healing, inflammation and vascular biology. Int. J. Biochem. Cell Biol. 39, 505-528.

Bernfield, M., Gotte, M., Park, P.W., Reizes, O., Fitzgerald, M.L., Lincecum, J., and Zako, M. (1999). Functions of cell surface heparan sulphate proteoglycans. Annu. Rev. Biochem. 68, 729-777.

Bouchard, J.F. and Lamontagne, D. (1999). Mechanisms of protection afforded by cyclooxygenase inhibitors to endothelial function against ischemic injury in rat isolated hearts. J. Cardiovasc. Pharmacol. 34, 755-763.

Bruegger, D., Jacob, M., Rehm, M., Loetsch, M., Welsch, U., Conzen, P., and Becker, B.F. (2005). Atrial natriuretic peptide induces shedding of endothelial glycocalyx in coronary vascular bed of guinea pig hearts. Am. J. Physiol. Heart Circ. Physiol. 289. H1993-H1999.

Chappell, D., Jacob, M., Hofmann-Kiefer, K., Bruegger, D., Rehm, M., Conzen, P., Welsch, U., and Becker, B.F. (2007). Hydrocortison preserves the vascular barrier by protecting the endothelial glycocalyx. Anesthesiology 107, 776-784.

Henry, C.B. and Duling, B.R. (2000). TNF- $\alpha$ increases entry of macromolecules into luminal endothelial cell glycocalyx. Am. J. Physiol. Heart Circ. Physiol. 279, H2815-H2823.

Jacob, M., Bruegger, D., Rehm, M., Welsch, U., Conzen, P., and Becker, B.F. (2006). Contrasting effects of colloid and crystalloid resuscitation fluids on cardiac vascular permeability. Anesthesiology 104, 1223-1231.

Jacob, M., Bruegger, D., Rehm, M., Stoeckelhuber, M., Welsch, U., Conzen, P., and Becker, B.F. (2007). The endothelial glycocalyx affords compatibility of Starling's principle and high cardiac interstitial albumin levels. Cardiovasc. Res. 73, 575-586.

Luft, J.H. (1966). Fine structures of capillary and endocapillary layer as revealed by ruthenium red. Fed. Proc. 25, 17731783.

Myler, H.A. and West, J.L. (2002). Heparanase and platelet factor-4 induce smooth muscle cell proliferation and migration via bFGF release from the ECM. J. Biochem. (Tokyo) 131, 913-922. 
Nieuwdorp, M., van Haeften, T.W., Gouverneur, M.C., Mooij, H.L., van Lieshout, M.H., Levi, M., Meijers, J.C., Holleman, F., Hoekstra, J.B., Vink, H., et al. (2006). Loss of endothelial glycocalyx during acute hyperglycemia coincides with endothelial dysfunction and coagulation activation in vivo. Diabetes $55,480-486$.

Pries, A.R. and Kuebler, W.M. (2006). Normal endothelium. Handb. Exp. Pharmacol. 1, 1-40.

Pries, A.R., Secomb, T.W., and Gaehtgens, P. (2000). The endothelial surface layer. Pflüger's Arch. 440, 653-666.

Rehm, M., Haller, M., Orth, V., Kreimeier, U., Jacob, M., Dressel, H., Mayer, S., Brechtelsbauer, H., and Finsterer U. (2001). Changes in blood volume and hematocrit during acute preoperative volume loading with $5 \%$ albumin or $6 \%$ hetastarch solutions in patients before radical hysterectomy. Anesthesiology 95, 849-856.
Rehm, M., Zahler, S., Lotsch, M., Welsch, U., Conzen, P., Jacob, M., and Becker, B.F. (2004). Endothelial glycocalyx as an additional barrier determining extravasation of $6 \%$ hydroxyethyl starch or $5 \%$ albumin solutions in the coronary vascular bed. Anesthesiology 100, 1211-1223.

Shih, G.C. and Hajjar, K.A. (1993). Plasminogen and plasminogen activator assembly on the human endothelial cell. Proc. Soc. Exp. Biol. Med. 202, 258-264.

van den Berg, B.M., Vink, H., and Spaan, J.A. (2003). The endothelial glycocalyx protects against myocardial edema. Circ. Res. 92, 592-594.

Vogel, J., Sperandio, M., Pries, A.R., Linderkamp, O., Gaehtgens, P., and Kuschinsky, W. (2000). Influence of the endothelial glycocalyx on cerebral blood flow in mice. J. Cereb. Blood Flow Metab. 20, 1571-1578.

Received July 9, 2007; accepted September 20, 2007 